\title{
Lightning Imaging with LOFAR
}

\author{
Olaf Scholten ${ }^{1,2, \star}$, Stijn Buitink ${ }^{3}$, Roxana Dina ${ }^{1}$, Qader Dorosti Hasankiadeh ${ }^{1}$, Wilfred Frieswijk ${ }^{4}$, \\ Freddie Hendriks ${ }^{1}$, Jeroen Muller ${ }^{1}$, Danny Sardjan ${ }^{1}$, Gia Trinh ${ }^{1}$, Antonio Bonardi ${ }^{5}$, Arthur \\ Corstanje $^{5}$, Ute Ebert ${ }^{6,7}$, Heino Falcke ${ }^{4,5}$, Jörg Hörandel ${ }^{5}$, Pragati Mitra ${ }^{3}$, Katharine Mulrey ${ }^{3}$, \\ Anna Nelles ${ }^{8}$, Jörg Rachen ${ }^{5}$, Laura Rossetto ${ }^{5}$, Casper Rutjes ${ }^{6}$, Pim Schellart $^{9}$, Satyendra \\ Thoudam $^{10}$, Sander ter Veen ${ }^{4}$, and Tobias Winchen ${ }^{3}$ \\ ${ }^{1}$ University of Groningen, KVI Center for Advanced Radiation Technology, 9747 AA Groningen, The Nether- \\ lands \\ ${ }^{2}$ Vrije Universiteit Brussel, Dienst ELEM, IIHE, Pleinlaan 2, 1050 Brussels, Belgium \\ ${ }^{3}$ Astrophysical Institute, Vrije Universiteit Brussel, Pleinlaan 2, 1050 Brussels, Belgium \\ ${ }^{4}$ Netherlands Institute for Radio Astronomy (ASTRON), 7990 AA Dwingeloo, The Netherlands \\ ${ }^{5}$ Department of Astrophysics/IMAPP, Radboud University, P.O. Box 9010, 6500 GL Nijmegen, The Nether- \\ lands \\ ${ }^{6} \mathrm{CWI}$, Centrum Wiskunde \& Informatica, Amsterdam, The Netherlands \\ ${ }^{7}$ TU/e, Eindhoven University of Technology, Eindhoven, The Netherlands \\ ${ }^{8}$ Physics and Astronomy, University of California, Irvine, CA 92697-4575,U.S.A \\ ${ }^{9}$ Department of Astrophysical Sciences, Princeton University, Princeton, NJ 08544, USA \\ ${ }^{10}$ Department of Physics and Electrical Engineering, Linnéuniversitetet, 35195 Växjö, Sweden
}

\begin{abstract}
We show that LOFAR can be used as a lightning mapping array with a resolution that is orders of magnitude better than existing arrays. In addition the polarization of the radiation can be used to track the direction of the stepping discharges.
\end{abstract}

\section{Introduction and summary}

Lightning is a familiar phenomenon, but still far from being understood [1]. There are recent suggestions for the mechanism that initiates lightning discharges [2, 3] as well as some qualitative understanding of how a lightning leader discharge propagates over distances of kilometers, but a quantitative theory is lacking. In particular, it is known that leaders with an excess of negative charge (called negative leaders) often proceed in a step-wise fashion, but the physical mechanism for this stepping is not understood. While stepping the leader emits strong radio pulses that can be used to locate its position in the sky. These radio pulses are used by Lightning Mapping Arrays (LMAs) to map the progress of leader development [4]. This makes a LMA the ideal instrument to study the physics involved in Lightning initiation and propagation.

Here we show that a new observation mode at LOFAR, a new generation radio telescope [5] build mainly for astronomy observations, allows for LMA observations with a resolution that is orders of magnitude better than presently existing LMAs, thus allowing for lightning observations with unprecedented resolution. The polarization of the signal can be used to reconstruct the direction of the

^e-mail: Scholten@KVI.nl 
discharge. Combining these we can construct a 4-D vector map of the discharge process with high precision. To interpret the observations we have developed a model for calculating radio emission from a stepped leader. In Ref. [6] it was reported that the radio pulses have a fine structure on the order of $10 \mathrm{~ns}$ which we have confirmed in our preliminary measurements.

Recently we have developed a non-invasive method for determining the electric-field in thunderclouds [7, 8] by measuring at the core of LOFAR the radio footprint from cosmic-ray air showers. It is of much interest to combine this with the LOFAR-LMA observations.

\section{State-of-the-art LMA}

Nowadays there are several Lightning Mapping Arrays (LMA) [4] in operation. To determine the position in the atmosphere of the source that emitted the radio signal one measures the time of arrival (ToA) in a network of antennas on the ground. To reach sufficient accuracy they are spread over distances of typically $60 \mathrm{~km}$ [9]. Most arrays operate in an abandoned TV broadcasting frequency band in the lower VHF band, $60-66 \mathrm{MHz}$ where the signal is digitized at a frequency of $25 \mathrm{MHz}$. The data is analyzed in blocks of $80 \mu$ seconds, called "Windows", in which the arrival time of the strongest peak is determined. The window size limits the maximum number of pulses to be analyzed to 12,500 per second. In each Window the arrival time is determined with a precision of $40 \mathrm{~ns}$ set by the resolution of the digitization. The relative timing of individual antennas is set using GPS. This has resulted in detailed maps of leader development that have contributed much to a better understanding of the physics of lightning, see [1].

\section{LOFAR}

The Low Frequency Array [5] (LOFAR) is a digital radio telescope that is primarily build for astronomy observations, consisting of many thousands of antennas. The antennas are grouped in stations, where each core station has 96 Low Band Antennas (LBA) and 48 High Band Antennas (HBA). Each LBA consists of two inverted V-shaped dipoles labeled X and Y. The LBA are sensitive to the frequency range of $10-90 \mathrm{MHz}$ and are used for the observations discussed in this work. The sensitivity range of the HBA is $110-240 \mathrm{MHz}$. The signals are sampled at $200 \mathrm{MS} / \mathrm{s}$ leading to a sampling time of $5 \mathrm{~ns}$. The approximately $2 \mathrm{~km}$ diameter LOFAR core contains 24 such stations with the highest density offered by the six stations located in a $320 \mathrm{~m}$ diameter region called the 'Superterp' near Exloo in the north of The Netherlands. Remote stations may lie at a distance of $1000 \mathrm{~km}$ from the core.

All antennas of LOFAR are equipped with ring buffers, that store the raw voltage traces of each individual antenna in the array for up to $5 \mathrm{~s}$. When a trigger is received, the ring buffers are frozen and their contents are copied over the network to a central storage location. In the observation mode pertinent to this work the trigger is generated when there is lightning activity.

\section{LOFAR as LMA}

At each step of a lightning leader a sizable current flows in short time. By the nature of this process, strong linearly polarized radio-frequency pulses are emitted at meter wavelengths, making LOFAR an exquisitely suited instrument for measuring them with the LBA. This is corroborated by model calculations [10] showing the the angular distribution of the emitted radiation as well as the polarization can be used to determine rather accurately the direction of the discharge step. In addition it is possible to extract from the shape of the measured pulse the time profile and the length scale over which the current flows. 
Data were taken with LOFAR-LBAs, in the 30-80 MHz band on June 19, 2013, while a thunderstorm was active at a distance of some $50 \mathrm{~km}$ from the telescope. The time trace of the LOFAR antennas on the Superterp as well as other stations up to distances of $30 \mathrm{~km}$ were analyzed.
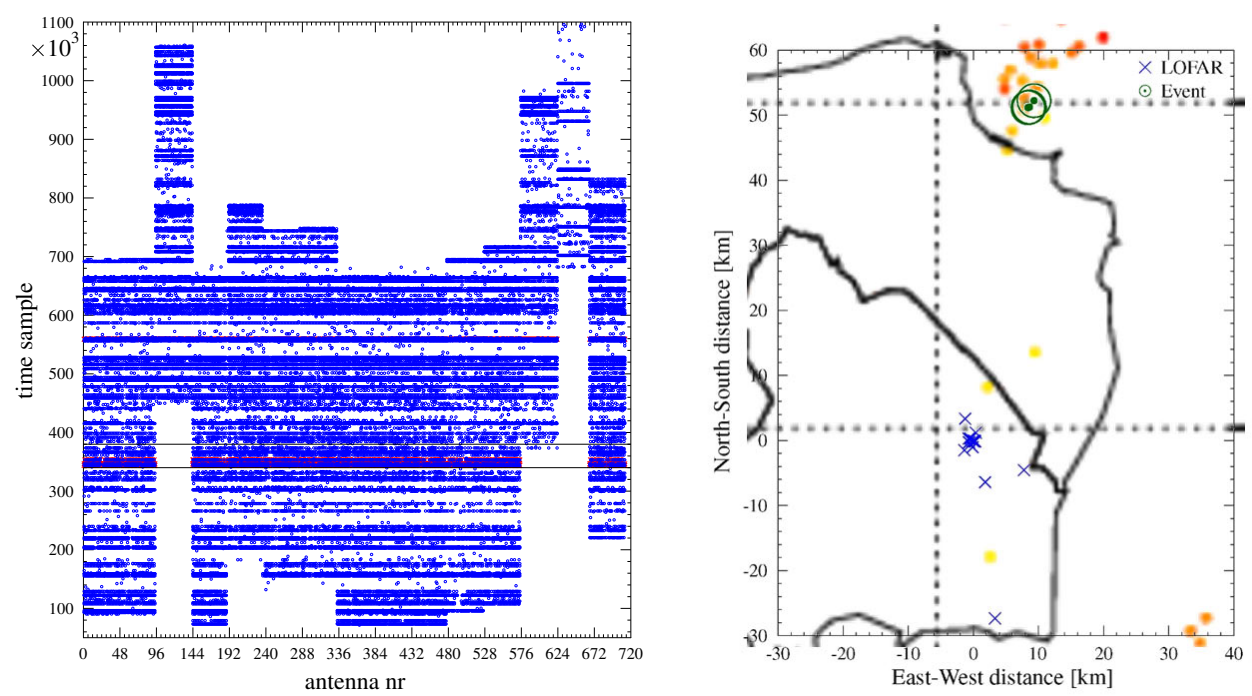

Figure 1. Left side shows pulse timing (vertical axis), indicated by dots, for all antennas (antenna number on horizontal axis) that were included in the present measurement. The traces of different stations (of $3 \mathrm{~ms}$ length, sampling time of $5 \mathrm{~ns}$ ) have been time-shifted to align the strongest peak in the time-sequences. One trace could not be aligned. The pulses marked in red for three events are analyzed for their source location as shown on the right overlayed on a map of the North-East corner of The Netherlands showing the borders of the provinces of Groningen and Drenthe. The orange dots indicate lightning observations by the Dutch weather service. The positions of the LOFAR stations that are involved in the measurements are indicated by blue crosses.

As the first step in the analysis of the data the pulse-patterns seen in the different stations are roughly lined-up as shown in Fig. 1 for the different stations. A pulse is labeled as such when, after Radio-Frequency Interference (RFI) mitigation, the power exceeds a certain threshold. This lining-up is necessary as for these older data the precise timing off-sets of the stations were not available. As can be seen this works very well for all 15 stations with the exception of one for which the off-set exceeded the trace-length and no matching could be made. Each station consists of 48 antennas spread over an area with a diameter of about $30 \mathrm{~m}$ for which the relative timing is known accurately. For the three events marked in red in Fig. 1 the source location and emission time is found by fitting the arrivaltimes of the pulses for each separate antenna adjusting the station offsets, but keeping them the same for all events. The fit reproduces the measurements with an accuracy of about 1 time sample. At the right panel of Fig. 1 the source locations (green circled dots) are overlayed with a map of North-East Netherlands showing the provinces of Groningen and Drenthe. The orange and red points label the positions of observed lightning discharges in an half-hour time slot around the LOFAR observations as determined by the Dutch weather service showing a good agreement with the found source locations.

As an example the Hilbert envelopes of a short section of 200 samples of the time traces for one particular event is shown in Fig. 2 for several stations in separate plots for each of the two polarizations. The Hilbert envelope is averaged over the antennas of a station for a specific polarization. This shows that the time traces for different stations are very similar and thus that the structure is genuine, not due to interference with random noise. It also shows that the time traces for the two polarizations 

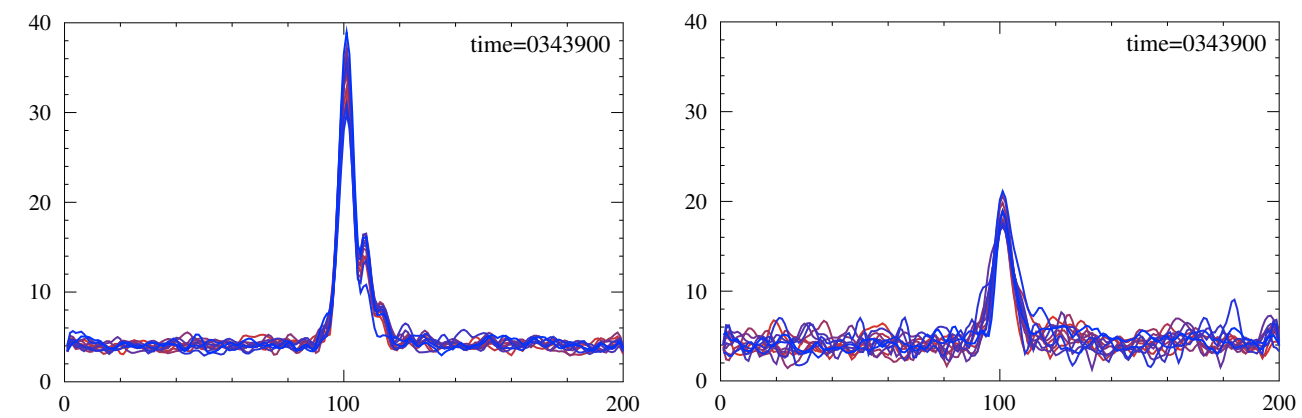

Figure 2. The average pulse shapes (Hilbert envelope), i.e. the average of the signals measured by the antennas in each station normalised to the integral in the $\mathrm{X}$ axis, (a different color for each station) for the two polarization directions (left and right hand sides). The total trace length shown is 200 samples of 5 ns, i.e. $1 \mu$ s.

are clearly distinct with a clear polarization-dependent structure for the pulse. Because the pulse is weaker in one polarization it is over threshold in fewer antennas giving rise to a more noisy signal. It should be noted that for some other events a much more complicated pulse structure is observed.

Work is still ongoing to determine the relative accuracy of source positions. In addition for this initial analysis [11, 12] the pulse timings were determined in single-sample precision. In the next step we refine this using pulse interferometry with which we should easily be able to obtain nanosecond timing accuracy.

\section{References}

[1] J.R. Dwyer and M.A. Uman, Phys. Rep. 534, 147 (2014).

[2] A. Gurevich, A. Karashtin, Phys. Rev. Lett 110, 185005 (2013),

[3] Anna Dubinova, et al., Phys. Rev. Lett 115, 015002 (2015).

[4] Some links to Lightning Mapping Array, Oklahoma LMA; SPORT, New Mexico; Ebro Valley;

J.D. Hill, et al., J. of Geoph. Res.: Atm 118, 8460 (2013)

[5] M. P. van Haarlem, et al., A5562013A2; arXiv:1305.3550; LOFAR Wikipedia page.

[6] N. Murray, P. Krider, J. Willett, Atmos. Res. 76, 455 (2005).

[7] P. Schellart et al., Phys. Rev. Lett 114, 165001 (2015), arXiv:1504.05742.

[8] G. Trinh, O. Scholten, et al., Phys. Rev. D 93, 023003 (2016), arXiv:1511.03045

[9] R.J. Thomas et al., J. Geophys. Res. 109, D14207 (2004).

[10] Freddie Hendriks, Bachlor thesis, University of Groningen, 2016.

[11] Roxana Dina, Master thesis, University of Groningen, 2014.

[12] Danny Sardjan, Bachelor thesis, University of Groningen, 2016. 